\title{
Modern Gas Sensors Allow Innovation
}

\author{
Maximilian Fleischer, \\ Siemens AG, Corporate Technology \\ Otto-Hahn-Ring 6, D-81739 München
}

\begin{abstract}
Gas sensors became a mature technology now and will be the base for innovations in industrial fields like building technology, medical, automotive, consumer and industrial technology. Modern gas sensors are designed in a Microsystems (MEMS) approach to leverage the integration of a complex functionality into a small-sized and low-cost device. Variations in the design of gas sensing Microsystems may yield a wide variety of device characteristics that can be exploited to fulfill heterogeneous application requirements. This can be done by using some variety of operating principles including ones not used so far. For successful industrialization, of a gas sensing application, care has to be taken in advance, that the chosen sensing principle has the potential to fulfill all needs of the intended application.

Examples are discussed. Gas sensors based on the conductance modulation of semi-conducting metal oxides are characterized by very simple and robust constructions. Limits are in selectivity. The successful applications for regulation of home gas burners and for Ethanol detection in human breath for car interlocks are discussed. The usage of the work function readout of gas sensing materials with FET-devices allows access to a wide variety of sensing materials that distinctly opens up the variety of gases that can be detected using metal oxide gas sensors. The usage of these gas sensors for room air quality and medical applications in the human breath are discussed. Optical gas detection in the NIR using the spectroscopy with Tunable Laser Diodes constitutes the high-end of gas sensing, yielding selective and high precise gas measurements
\end{abstract}

\section{Introduction}

Following a statement attributed to P. Bamelis, "Innovation" is "the transfer of knowledge to money". For industrial organizations this means that new technologies are taken up to implement competitive products on the market, which is needed to ensure the own survival amongst the fittest. Gas sensors became a mature technology now, that open the access to a variety of innovations in the building, medical, automotive, consumer and industrial market segment. Modern gas sensors are characterized by improved functionality to fulfill the applications requirements. It has to be stated that there is no unique operation principle that can be used for all applications.

To allow innovation and achieve success in the market on the long-term perspective, industry has to focus to a few selected sensing platforms, each of them having a market potential that is large enough to allow cost-effective manufacturing of the sensors by the sensor producer. Stable business models usually rely on a structure of one component manufacturer for the sensing element platform; he delivers his sensors to a variety of sensor system manufacturers in different applications. Manufacturing of a sensor for only one application or one main customer usually imposes high risks and instability. The platform character of the sensing technology has to allow to adapt the sensor characteristics to several applications.

\section{Semiconducting metal oxide sensors}

The principle of the classical semiconductor gas sensors is well known since the onset of the commercialisation of the classical "Taguchi-type" sensors. A semi conducting metal oxide, typically $\mathrm{SnO}_{2}$, is heated to temperatures of several hundred ${ }^{\circ} \mathrm{C}$ to become conductive. Due to gas interactions, the conductivity changes reversibly with the partial pressure of reactive gases in the ambient. There is deep knowledge on that, which is described e.g. in [1,2]. These sensors are characterised by the need of certain heating power of several $100 \mathrm{~mW}$ to maintain the operating temperatures and by a response of the materials to all reactive (reducing or oxidising) gases present in the ambient - which might be advantageous or disadvantageous, dependent on the application. Significant advances towards the recognition of smell pattrens were made by combing several sensors an using statistical methods for signal evaluation, for the state of the art see [3]. Novel developments rely on a micromachined set-up,that provide for the possibility of quick 
temperature changes. This allows to reduce the power consumption and to increase the selectivity of the sensors by employing sophisticated signal evaluation using the temperature transient response [4]. The requirements for the absence of burn-in phenomena [5] and to the long-term stability of the gas sensitivity however even increase using this procedure

The contributions of the Siemens Corporate Technology focused on the development of new sensing materials like $\mathrm{Ga}_{2} \mathrm{O}_{3}, \mathrm{SrTiO}_{3}$ thin films or $\mathrm{WO}_{3} / \mathrm{TiO}_{2}$ mixed oxides. The common of all these materials is that they are chemically very robust compared to the classical $\mathrm{SnO}_{2}$ sensing material and are operated at higher temperatures. This allows the usage of these semiconductor gas sensors in chemically harsh conditions like industrial environments or exhaust gases [6]. An example is the use of the $\mathrm{Ga}_{2} \mathrm{O}_{3}$ sensors [7] in the exhaust gas of heater appliances, see fig. 1, where for the first time a reliable detection of unburned fuel components was made possible

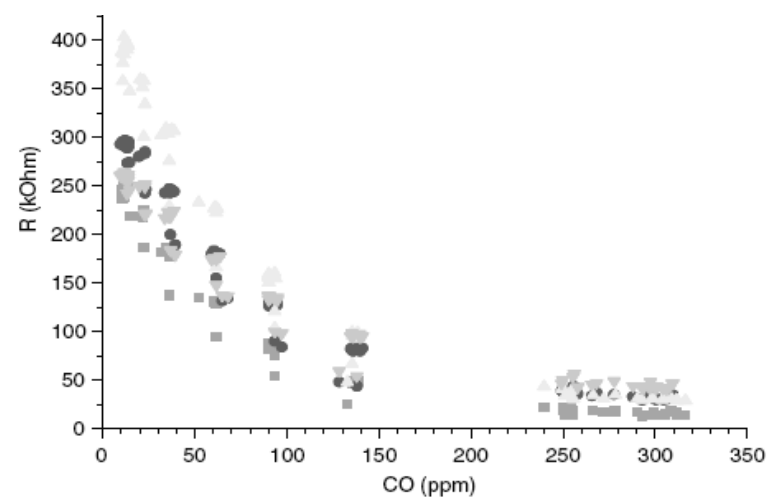

Fig. 1: CO-sensitivity of four different specimens of $\mathrm{Ga}_{2} \mathrm{O}_{3}$ sensors operated at $750^{\circ} \mathrm{C}$ in the exhaust gas of a pre-mix natural gas burner.

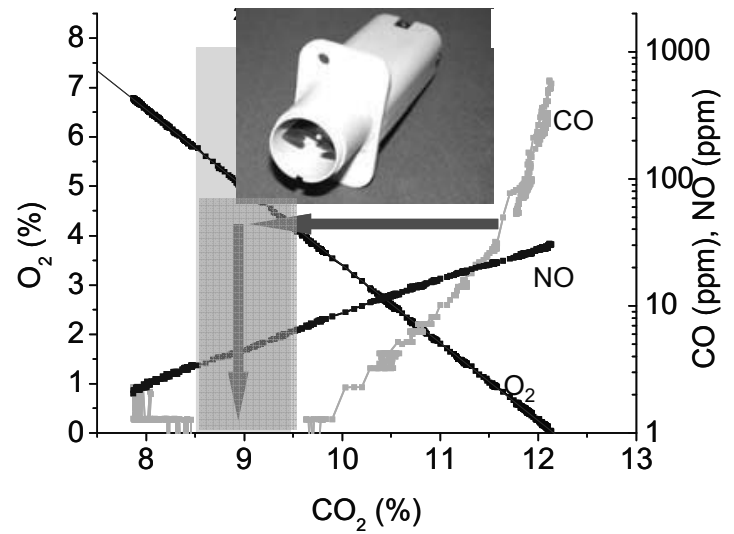

Fig. 2: Exhaust gas composition of a modern natural gas burner. The $x$-axis is gas/air ratio. The target range for burner regulation is highlighted.

Besides sensor stability in the application environment, a proper strategy on the usage of the sensing material has to be devised for the individual application. The sensor mainly detects unburned natural gas residues like $\mathrm{CO}, \mathrm{H}_{2}$ in the range of a few tens of ppms. In the optimal combustion region (highlighted, $\mathrm{CO}_{2}$ about 9\%). The concentration of the target gases in this range is too low to be detected, see fig. 2. The solution, like in many other applications, can be found in the inclusion of system knowledge: when an excess of natural gas is injected, the CO-value rises to a detectable concentration range. At the state where a certain threshold of $\mathrm{CO}$ is detected by the sensor, the control reduces the natural gas feeding by a defined value, which results in a jump to a combustion regime at the optimal value. This has already been implemented in heating systems of houses in large scale. These sensors will provide a small and cost-effective substitution for the conventional $\lambda$-probe in small motors in the future.

Another application is the detection of an ethanol threshold in human breath, usable for car interlocks as well as to protect drunken people from operating dangerous devices. People exhale into a device equipped with an ethanol sensor. This device has to be equipped with an intelligence to monitor the onset of exhalation. The reading of the sensor is done after a defined exhalation volume when air from the lung is present (alcohol from the mouth cavity may cause false readings). To avoid disturbing effects by cooling of the sensor, special measurement chamber geometry needs to be used., see figs. 3 and 4 . Electrochemical cell based solutions are too expensive for consumer solutions here.

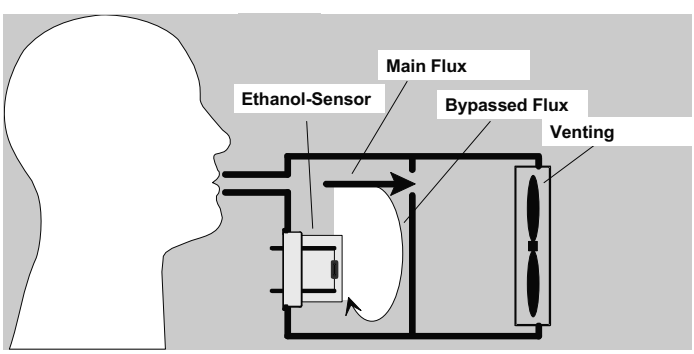

Fig. 3: For exhaled air measurement, a geometry is used that feeds only part of the exhaled breath to the sensor to avoid thermal effects but ensures rapid equilibrium.

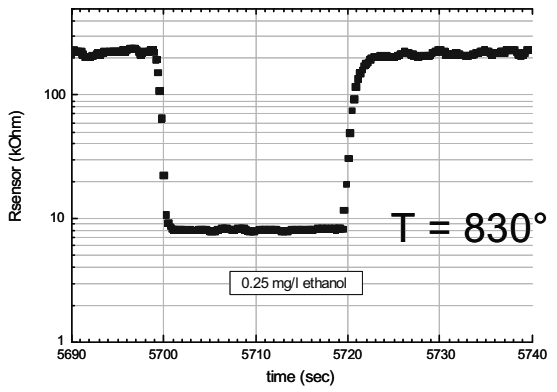

Fig. 4: Quick reaction of sensor to changing ethanol concentrations. 
Usually the sensor must be switched off so save battery power. When the device is switched on, only very few seconds are allowed for the sensor to reach a stable baseline. This is given by a miniaturized $\mathrm{Ga}_{2} \mathrm{O}_{3}$ sensor with chip dimensions below $1 \mathrm{~mm}$, which very quickly attains chemical equilibrium since it is operated at temperatures above $800^{\circ} \mathrm{C}$, see fig 5 . Low sensitivity to metabolic by-product like acetone is essential to avoid false readings.

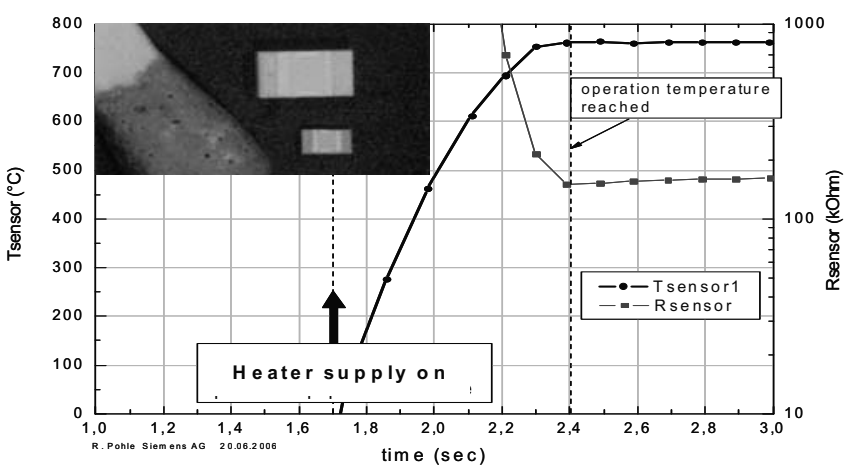

Fig. 5: Miniaturized version of the $\mathrm{Ga}_{2} \mathrm{O}_{3}$ sensor compared to the tip of a match and its power up behavior.

A high stability of the baseline resistance in this material is due to its conduction mechanism, where the carrier mobility is independent on the grain boundaries of the sensing material [8].

\section{Sensors using work function readout}

Sensors based on heated metal oxides use a current that has to pass through different barriers (grain boundaries, metal electrodes). All these influences add to the original gas sensitive effects that arise due to the surface reaction of the gas with the sensitive material. This makes sensors complicated to understand and often quite sensitive to side effects. The situation where an electrical potential that arises due to the gas adsorption at the sensitive material (see Fig 6) is directly used as gas sensitive effect, is easier to control. The size of these surface potentials or work function changes is in the order of $10-100 \mathrm{mV}$. A new generation of gas sensors uses the change of the work function which is read-out by a FET [8].

The pioneers in the construction of such type of set-up were I. Eisele and Th. Doll [9], who used a hybrid approach with one part being a gateless FET and the other a substrate equipped with a sensing film, both brought together to form a gas sensor. To make such things industrially usable, Siemens, together with Micronas developed a flip-chip variant of this device that allows precise mounting as well as the usage of low-processing temperatures. [10]. A sensing material free of choice is deposited on a flat carrier substrate forming the later gate-electrode. The Si FET-chip is separately prepared in standard CMOS. Finally both parts are brought together so that a defined air gap is formed, and are fixed.

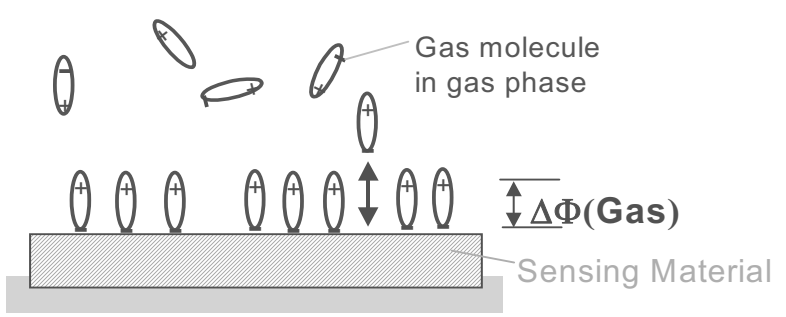

Fig. 6: Potential at the surface of a gas sensitive material due to orientated molecule adsorption $(\uparrow)$

Fig 7: Schematics of a sensor where the change in work function of the sensing layer capacitive couples to the read-out FET

$(\rightarrow)$

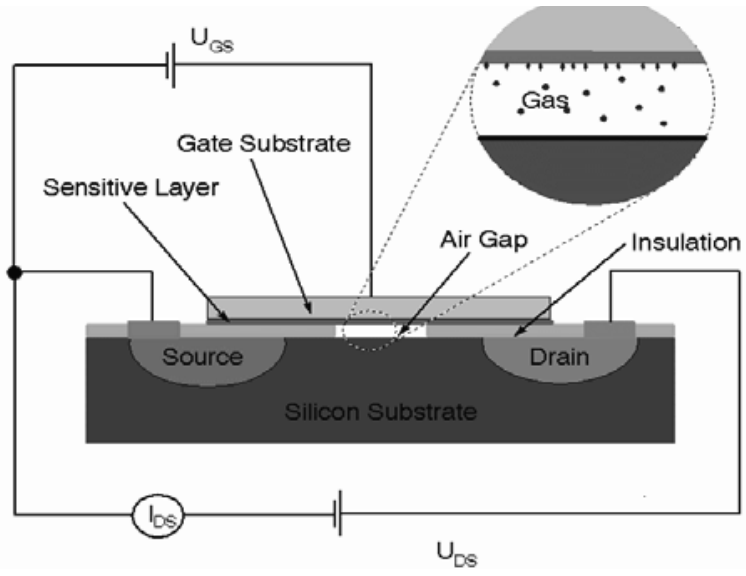

These sensors are characterized by an

- $\quad$ unprecedented freedom in the choice of sensing materials. The gas receptor does not need to be an oxide. All organic molecules, polymers, metals, salts may be used in this sensing platform.

- $\quad$ No heating is necessary; the sensors can be operated at room temperature.

- Multiple readout channels can be realized on chip, allowing for small sensing arrays. 


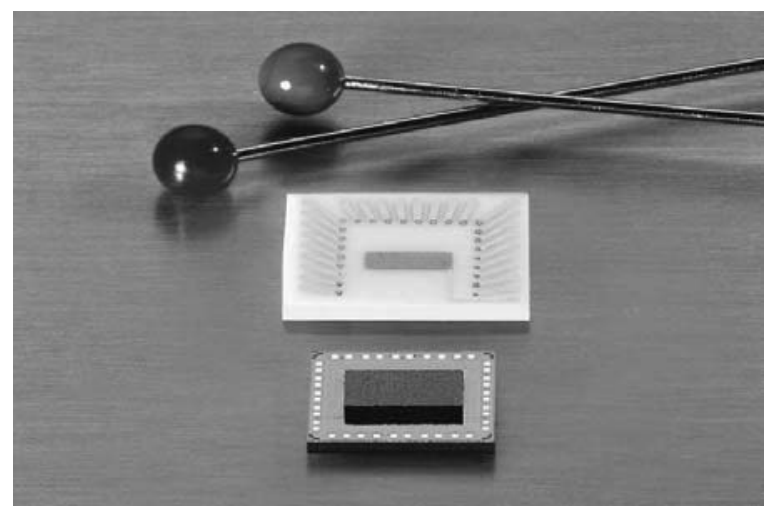

Fig. 8: Sample of a GasFET in comparison to needles. The lower part shows a sensor which consists of the Si readout chip done in CMOS technology and the small carrier of the sensing layers which is fixed above the Si-chip. The sensing layer is at the bottom of the carrier and facing the FET-channel.

Dependent on the material of the gas receptor layer this sensors may be operated at room temperature or may be operated with a heating element integrated in the Silicon with temperatures up to $150^{\circ} \mathrm{C}$

The choice of the materials allowed the development of a wide range of gas sensitive layers, including materials for gases like $\mathrm{NO}_{2}, \mathrm{NH}_{3}$, r.h., $\mathrm{H}_{2}, \mathrm{O}_{3}, \mathrm{HC}, \mathrm{CO}, \mathrm{CO}_{2}$ and solvents [8]. The example of $\mathrm{CO}_{2}$, which was previously hard to be detected with a solid state gas sensor is shown in fig 9 . In this case sensing layers are made from $\mathrm{BaCO}_{3}$ or from $\mathrm{BaTiO}_{3} / \mathrm{CuO}$ mixed oxides.

The application of these devices will allow the regulation of climatisation systems in building as well as in cars. Only when the local ambient deteriorates, ventilation is applied. The output is a considerable energy saving in both cases. It has to be noticed that the power consumption for the heating/ventilation/ AC of a building is about $40-60 \%$ of the overall energy expense to run the building. On-demand ventilation could cut this part of the energy expense to the half. To judge the air quality, three main parameters need to be measured, which are the

$$
\text { the } \mathrm{CO}_{2} \text {-content, the humidity, as well as smells. }
$$

Due to the versatility of the gas sensing layer of the GasFET platform, sensors these three parameters could be implemented in one solid state sensor for the first time without having to use expensive optical $\mathrm{CO}_{2}$ detection.
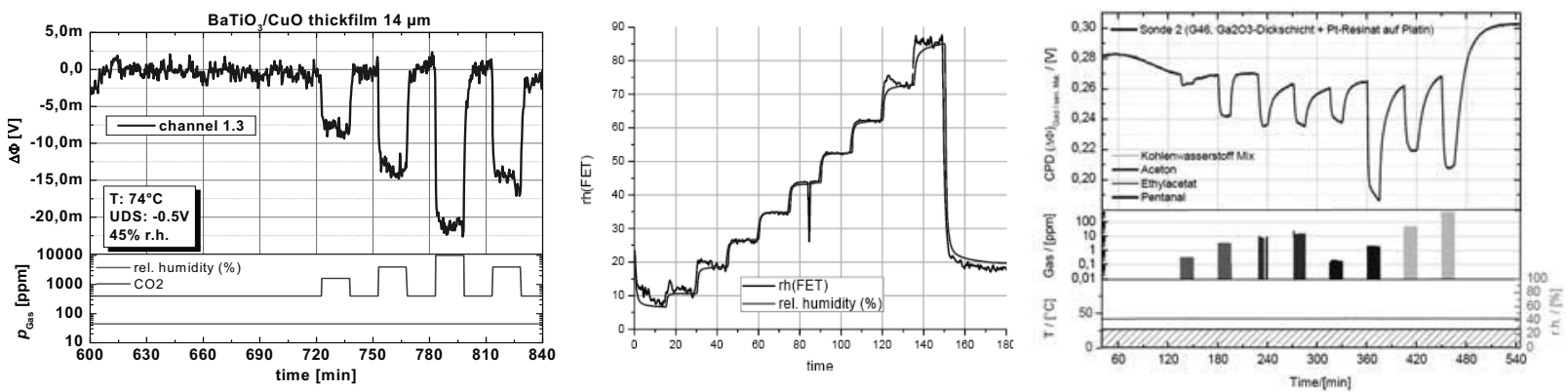

Fig. 9: Detection of $\mathrm{CO}_{2}$ (changes from 350ppm, to 1000/3000/10000ppm), relative humidity in comparison to a reference humidity sensor and of "smell" (VOCs, Acetone, Ethylacetate, Pentanale) [11] with the GasFET.

Another application which is accessible a moderate costs with this sensing platform relates to medical technology. Human breath contains some trace gases which correlate to diseases. One prominent example here us the acetone content of the human breath that increases in the case of abnormal blood glucose values. However the correlation turned out to be too weak to allow a reliable prediction of the blood glucose level based on an acetone analysis.

A medically validated example for a gaseous biomarker is the NO-level in the human breath. A rise of NO from 10-30, which constitutes the individual "normal" level to about $100 \mathrm{ppb}$, indicates an inflammatory state of the lung that may lead to an asthma attack. GasFET equipped with CuPhthalocyanine layers [12] can detect this rise. The patient gets warned that an asthma attack is likely to occur, has sufficient time to adjust the dose of cortisone so can prevent asthma attacks. The issue for the sensing technology here is the sensitivity as well as the selectivity of the sensing layer. CuPhthalocyanine reacts selectively to $\mathrm{NO}_{2}$. So a conversion step has to be taken that quantitatively oxidizes the $\mathrm{NO}$ to $\mathrm{NO}_{2}$. Optimized $\mathrm{Cu}$-Phthalocyanine layers have shown to be able to detect the resulting $\mathrm{NO}_{2}$ with sufficient sensitivity, see fig. 10 as well as selectivity, see fig. 11 . 


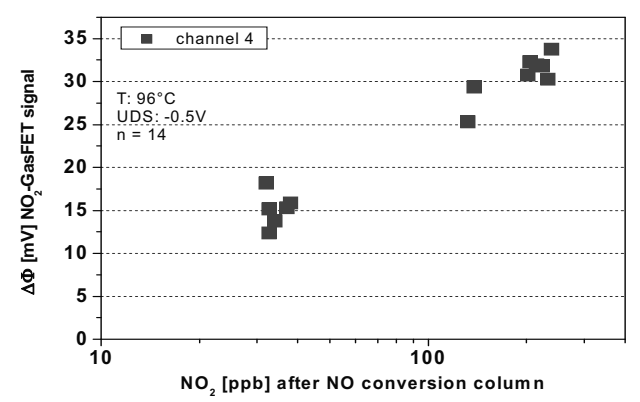

Fig. 10: Detection of NO in human breath in the 30ppb level (OK) and >100ppb level (Not-OK). Before the detection the $\mathrm{NO}$ was converted to $\mathrm{NO}_{2}$.

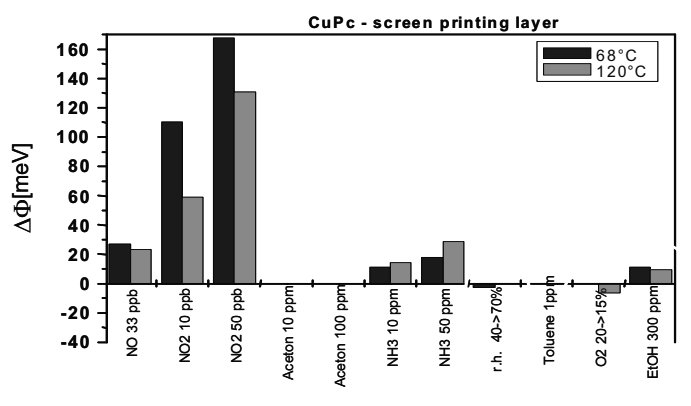

Fig. 11: Selectivity of $\mathrm{NO}_{2}$ sensing towards interferences in human breath as well as changing humidity.

\section{Spectroscopy of adsorption lines of gases}

This technology is by far the most precise, however also somewhat cost-full method to detect gases. The optical absorption, especially in the near-infrared range, of simple gas molecules are characterized by single adsorption lines that are grouped in so-called vibrational-rotational bands. The adsorption lines by themselves are a characteristic optical fingerprint of a gas species. Their with is so small $(0.1$ $\mathrm{nm}$ ), so that it is usually possible to find a spectral region where only the target has an optical adsorption. With the NIR laser diodes (DFB or VCSEL type) small light source are available that can be wavelength tuned within a few to several $\mathrm{nm}$. These diodes do not require the liquid $\mathrm{N}_{2}$ cooling as required by the former lead salt based diodes. Tuning can be done with several $100 \mathrm{~Hz}$ repetition rate.
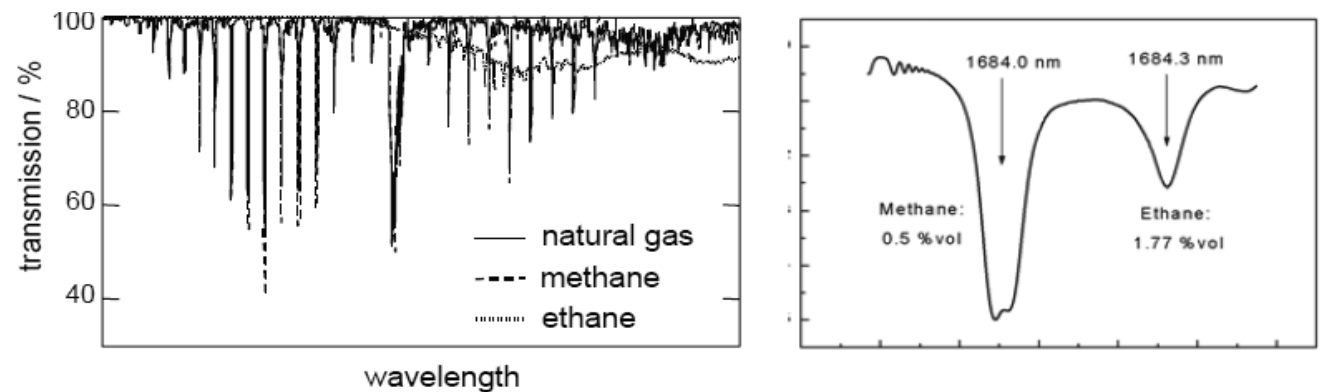

Fig. 11: Adsorption of natural gas (main components are methane and ethane) close to 1,6 $\mu$ m wavelength. The adsorption lines of both components are separated.

Gas concentrations can then be measured by an absorption measurement over a known optical path, see fig. 12. Often a light reflector is used to allow laser and receiver diode to be located close.

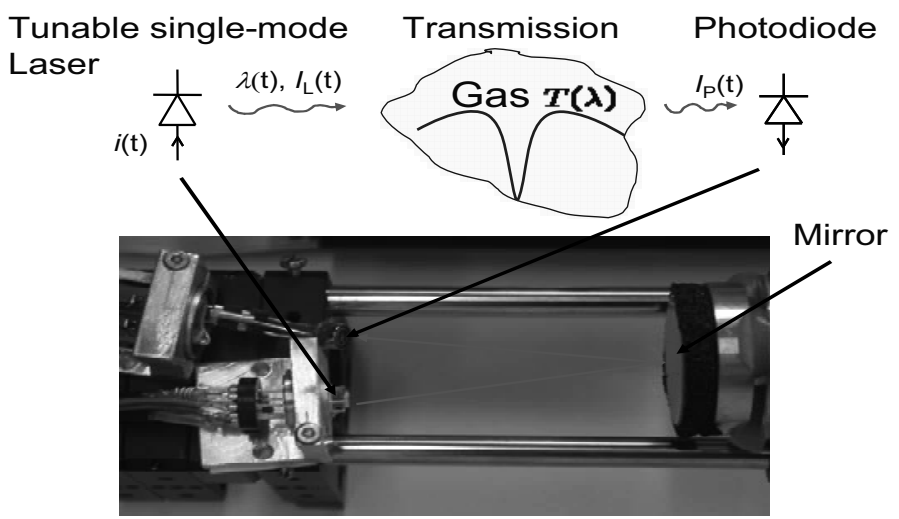

Fig. 12: Sensor set-up for spectroscopy of gas adsorption lines. The Laser diode emits the light which passes cavity containing the gas mixture to be evaluated, is reflected by a mirror and collected by an NIRphotodiode.

The light absorption is governed by the Lambert-Beer law, so partial pressure of the gas can be calculated from the attenuation of the light intensity.

The advantage of this technique over conventional optical absorption is threefold

- Since the wavelength tunability of the diodes is large compared to the with of the emission line, a signal with no as well as with maximum absorption is generated. The ratio of these two values is evaluated. As a consequence a total loss of light (degradation of optical components or dirt on the optical windows) which similarly influences both values does not influence the sensor signal.

- The measurement can be made gas selective: since the single absorption lines are so narrow, spectral regions can be found where there is no competing absorption by another gas. 
- In several cases the tuning range (especially with VCSEL type diodes) is sufficiently large to cover absorption lines from two gases, allowing to detect two gases with one diode.

Several applications have been realized with this technology. An example is natural gas. Close to 1,6 $\mu m$ wavelength there are adjacent absorption lines of methane and ethane that can be attained with one diode. One application is to distinguish natural gas (ethane present) from methane generated by bio-fouling processes (no ethane). The other is to quantify the main components of natural gas to have a reliable estimate of the calorific value. Monomodal laser diodes with larger wavelength (2 to $2,5 \mu \mathrm{m})$ become increasingly available, allowing access to stronger adsorption line und thus to lower gas concentrations. One milestone here was the recent breakthrough by the demonstration of ppm resolution for CO using a 2,3 $\mu \mathrm{m}$ VCSEL laserdiode and an optical path of only $2 \times 5 \mathrm{~cm}$.

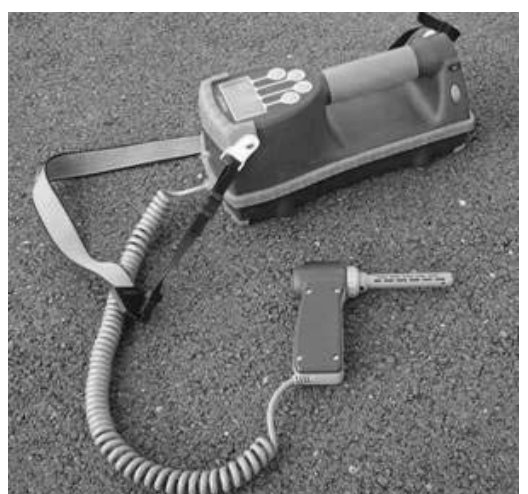

Fig. 13: Professional device for Natural gas leakage detection. The optical path is in the handle and allows for $10 p p m$ resolution

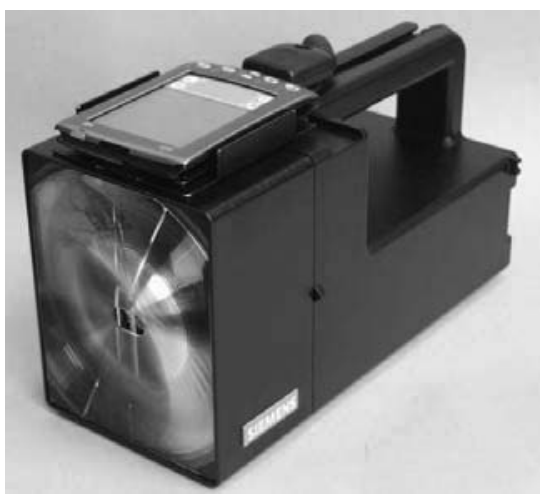

Fig. 14: Remote detection of Natural gas leakages using a "Laserpointer"-geometry. The light beam is diffusely reflected at some surface and a Fresnell lens collects backscattered light.

This measurement technique is by far the most accurate for gas measurements in terms of precision, selectivity and baseline stability. However, the prices of the laser diodes typically range from three to the four digit range in $€$. As a consequence this technology is mainly used for industrial applications. However it has to be noticed that the prices for the laser diodes currently decrease since manufacturing volumes of these diodes increase.

Several new technologies are currently coming up, that promise innovations in additional applications. Amongst the most promising are catalyst determined mixed potential sensors [15], new structural approaches optimizing selectivity in oxides [16] and the employment of the gas sensitive behavior of direct thermoelectric effects [17].

\section{REFERENCES}

[1] N. Barsan, U. Weimar, Journal of Electroceramics 7, 143.167, 2001

[2] D. Kohl, Journal of Physics D: Applied Physics34 (2001) R1-R25

[3] F. Rock, N. Barsan, U. Weimar, Chemical Reviews 108, 2 (2008) 705-725

[4] Z. Ankara, A. Schütze, Proc. EUROSENSORS 2008, Dresden, Germany, 7-10.9.2008, pp. 495498, ISBN 978-3-00-025217-4.

[5] P. Reimann, T. Conrad, H. Hettrich, A. Schütze, Sensoren und Messsysteme 2008, VDI Berichte \#2011, Hrsg. VDI Wissensforum GmbH, ISBN 978-3-18-092011-5, S. 539 - 548.

[6] U. Lampe, M. Fleischer, H. Meixner, Sensors \& Actuators B17 (3) (1994) 187

[7] www.steinel.ch

[8] M. Fleischer, Meas. Sci. Technol. 19 (2008)1-18

[9] B. Flietner, T. Doll, I. Eisele, Sens. Act. B, 22, 109-113, 1994

[10] R. Pohle, E. Simon, M. Fleischer, H. Meixner, H.-P. Frerichs, M. Lehmann, H. Verhoeven, Proc.TRANSDUCERS 2003, Volume 1, Issue , 8-12 June 2003 Page(s): 135 - 138 vol.1.

[11] S. Stegmeier, P. Hauptmann, M. Fleischer, Proc.. EUROSENSORS 2008, 7.-10. September 2008, Dresden, Germany, ISBN 978-3-00-025217-4

[12] U. Weimar, E. Simon, M. Fleischer, H.-P. Frerichs, Ch. Wilbertz and M. Lehmann, Sensors and Actuators B, 118 [1-2] (2006) 249-254

[13] O. Henning, R. Strzoda, E. Magori, E. Chemisky, Chr. Tump, M. Fleischer, H. Meixner, I. Eisele Sensors \& Actuators B 95 (2003)151-156

[14] J. Chen, A. Hangauer, R. Strzoda, M. Ortsiefer, M. Fleischer and M.-C. Amman, 21st Annual Meeting of The IEEE Lasers \& Electro-Optics Society, Newport Beach, CA | 9-13 November 2008

[15] D. Biskupski, K. Wiesner, J. Kita, M. Fleischer, and R. Moos, Sensor Lett. 6, 803-807 (2008)

[16] N. Barsan, D. Koziej and U. Weimar, Sensors and Actuators B 121 (2007) 18-35

[17] F. Rettig, R. Moos, IEEE Sensors Journal, 7, 1490-1496 (2007) 